\title{
West Nile Virus: Measures against Emergence in Malaysia
}

\author{
Mohammed MN and Yasmin AR* \\ Department of Veterinary Pathology and Microbiology, Faculty of Veterinary \\ Medicine, Universiti Putra Malaysia, Malaysia \\ *Corresponding author: Nor Yasmin binti Abd Rahaman, Department of Veterinary
}

Laboratory Diagnostics, Faculty of Veterinary Medicine, Universiti Putra Malaysia,

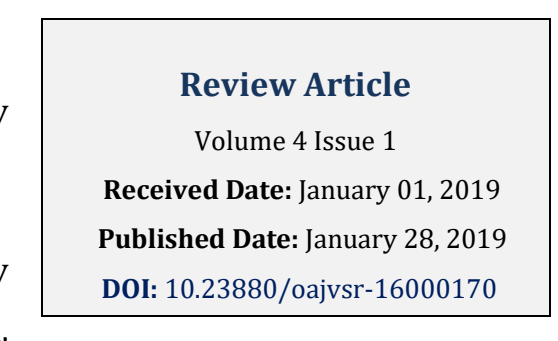
43400 UPM Serdang, Selangor, Malaysia, Tel: 03-86093473; Email: noryasmin@upm.edu.my

\begin{abstract}
West Nile virus has a potential to emerge in new areas and cause large epidemics as was witnessed in the United States following its introduction in 1999. The virus is now a global public health threat, having been detected on every continent except Antarctica. Once restricted to Africa, its expansion beyond its natural habitat is related to some viral, vectoral, anthropologic and environmental factors. The successful establishment and spread of the virus depend in part on viral adaptations, availability of competent hosts and mosquito vectors and suitable environmental conditions. A combination of measures can be applied to minimise the likelihood of a WN virus epidemic. Available vaccines are only for veterinary use, human vaccines are still in development. Vector control, animal vaccination, targeted surveillance and strong cooperation between relevant authorities are important in preventing a WN virus epidemic in Malaysia.
\end{abstract}

Keywords: Virus; Epidemics; Malaysia

\section{Introduction}

West Nile virus has a potential to emerge in new areas and cause large epidemics as was witnessed in the United States following its introduction in 1999 [1]. The virus is now a global public health threat having been detected on every continent except Antarctica [2]. Once restricted to Africa where it was first isolated in 1937 [3], its expansion beyond its natural habitat and establishment in new areas is related to some viral, vectoral, anthropologic and environmental factors. These include viral adaptations, availability of competent hosts and mosquito vectors and suitable environmental conditions. The rapid spread through the United States was a surprise and a cause for concern; the virus had not been reported in the Western Hemisphere before 1999 [4] and despite efforts to control its spread, it successfully did spread through the country and spilled northwards into Canada and southwards to South America [5].

Historically, WN virus circulated in Africa, the Mediterranean, the Middle East and parts of Europe, causing sporadic outbreaks in humans and horses. Kunjin virus is a strain of the virus endemic to Australia was isolated in Malaysia in 1970 [6]. The outbreak in New York was caused by a strain nearly identical to the one that caused an outbreak in Israel previously [7]. The means of its introduction is unknown and shows how easy an infectious agent from a distant area can get introduced and become well adapted to local conditions elsewhere. In Malaysia, reports of WN virus activity was first published in 1963 and later in 1970 [6,8]. More recently antibodies were detected in sera from captive birds in Selangor and from the Orang Asli population 


\section{Open Access Journal of Veterinary Science \& Research}

$[9,10]$. These clearly provide evidence of WN presence in Malaysia, though no clinic infections have been attributed to it in either humans or animals. Considering the potential of the virus to mutate and become pathogenic to humans and animals [11], it is important to monitor virus activity and control its spread before it causes an epidemic.

WN virus is a zoonotic flavivirus that naturally circulates between birds and Culex mosquitoes [12]. It can infect and cause disease in humans and a number of vertebrate species; these are however considered deadend incidental hosts since they are not capable of perpetuating virus transmission. The genome of $\mathrm{WN}$ virus is a single-stranded RNA $11 \mathrm{~kb}$ in size with a single open reading frame [13]. Mosquitoes acquire the virus following a blood meal from viremic reservoir hosts, which are several species of birds. The virus then replicates in the gut epithelium and eventually disseminates in the mosquito and replicates in the salivary gland. During blood meal on susceptible hosts, virus-containing saliva is secreted by the mosquito to prevent clotting, leading to infection.

About 3-14 days after infection a mild febrile illness is observed in about $20 \%$ of infected individuals. In humans, about $1 \%$ of infected individuals develop severe encephalitis as a result of the virus crossing the bloodbrain barrier. Symptoms include high fever, stupor, headache, coma, neck stiffness, disorientation, tremors, muscle weakness, convulsions and paralysis. Death may occur following these symptoms and treatment is supportive in both humans and horses. Vaccines are available for horses but non for humans. Vector control is very critical in the control of WN virus.

WN virus outbreaks are typically first noticed in animals before human infections are observed. Effective outbreak prevention and control measure will depend on rapid and accurate identification of illnesses due to the virus in sentinel animals and in patients. The abundance of both the vectors and reservoir host for $\mathrm{WN}$ virus in Malaysia is a challenge especially for preventing animal infections. Reducing the population of and exposure to the vectors are central to the success of any prevention and control strategy against $\mathrm{WN}$ virus.

WN virus transmission be can be interrupted by preventing or minimizing mosquito bites. This can be achieved by eliminating the vectors, reducing the vector population and by putting a barrier between mosquitoes and people. Understanding the life cycle and feeding behaviour of the Culex mosquito can help in designing effective vector control strategies including those directly targeted at the vector and others focused on the environment and human behaviour.

\section{Transmission Cycle of West Nile Virus}

WN virus naturally exists in a transmission cycle between birds and mosquitoes. Infected birds can have a high and sustained viremia compared to dead-end hosts like horses and humans. They are thought to be important in the introduction of the virus to new habitats, and the start of a WN virus epidemic is usually marked by death in birds.

Ornithophilic mosquitoes acquire the virus during blood meals on infected birds. The virus causes a persistent infection, replicating in the midgut epithelia and concentrating in the salivary glands, resulting in high virus titre in the saliva [14]. Susceptible hosts are infected by the mosquito's saliva when they are fed on.

Between its transmission from the birds to mosquitoes and other hosts, the virus constantly mutates through antigenic drifts, resulting in a diverse viral population. Through this constant change in its genome conformation, novel strains that are pathogenic and fit for transmission emerge, leading to outbreaks $[15,16]$.

\section{Life Cycle of WNV Vectors in Malaysia}

The life cycle of mosquitos typically has four developmental stages; adult, eggs, larval and pupal stages and generally takes between 13-15 days to complete. Mosquitoes breed in aquatic environments, where the female adult mosquitos lay floating eggs on water, each laying from 100 to 500 eggs. Culex eggs are cigar shaped and are laid in clusters. Swamps, puddles of water, tree holes filled with rain water are suitable for mosquitoes to lay their eggs in their natural environment. Closer to humans, clogged up gutters, heaps of trash, disposed cans and old discarded tyres all collect water in them and provides suitable breeding grounds for mosquitoes.

The eggs hatch into aquatic larvae in a few days. The larvae are submerged just beneath the surface and breathe through specialised structures. They feed and grow rapidly, undergoing 4 different moults in 3-5 days before they shade their larval skin and metamorphose to a pupa. During the pupal stage which lasts a day or two, feeding stops and development into adult mosquitoes in completed. The adults break out of the pupal skin, dry off 
its wings, waits for its exoskeleton to harden and then fly off to feed on nectar, juice or blood.

\section{Feeding Behaviour of Culex Mosquitoes}

Mosquitoes of the Culex genus have a preference for birds, but also feed on mammals including humans, and on amphibians, making them important in the transmission of WN virus between birds and to other animals $[17,18]$. Female Culex mosquitoes were found indoors where they seek blood meal in avian shelters, rabbit hutches and in urban dwellings where they may feed on humans, potentially transmitting arboviruses in the process [19].

Different mosquito species have different times for seeking a meal. Understanding this behaviour can help predict the most likely periods bites will occur and this can be used in behaviour modification to reduce contact with the mosquitoes. Time of the day and light can affect blood-seeking time of certain Culex species, however Culex pipiens seems to refractory to the effect of light and it will seek blood meals whenever a host becomes available [20].

\section{Strategies for Prevention and Control of WN Virus Transmission}

Effective mosquito control programmes require the use of different strategies systemically implemented to eliminate the source of the pathogen, reduce contact between the vector and the source of the pathogen, reducing the vector population and preventing/reducing contacts between the vectors and susceptible hosts. WN virus cycles naturally between birds and mosquitoes. Obviously, the practical approach will be to reduce the vector population thereby reducing contacts both with the reservoir host and dead-end hosts. Different approaches for the prevention and control of WN virus infection is discussed below.

\section{Surveillance for WN Virus}

WN virus has been detected in mosquitoes in regions where clinical infections had not been recorded in animals or human. Because the virus mutates and evolves quite easily, the virus can acquire traits that confer better fitness in hosts and greater virulence. WN virus outbreaks in a population begin in animals and human illness follows afterwards. As much as $80 \%$ of infected individuals will not develop any signs of infection. Early detection of the virus in birds, horses and other animals is important for an early detection of an outbreak. It is therefore important to establish a virus surveillance programme in mosquitoes and birds to determine the risks of a WN outbreak in the population.

\section{Vector Control}

Reducing the population of mosquitoes is beneficial in preventing the transmission of WN virus, other arboviruses and pathogens. The different stages of the mosquito life cycle could be targeted, but control of adult mosquitoes and larvae are important. Larva destruction has been shown to significantly reduce the adult populations of mosquitoes.

Control of adult mosquitoes: Over the years, many chemicals have been developed and used for the control and elimination of mosquito species around the world. The environmental impact of using these chemicals is high as it also destroys population of beneficial insects like the bees that are important pollinators. The use of insecticides indoors and environmental spraying of large areas around farms and human dwellings have been successful in keeping mosquito populations low, although these need to be combined with other measures for a sustained control. Asides the environmental impact of chemicals in mosquito control, resistance to pesticides is of concern in Culex species [21-23]. Monitoring for resistance should be done and it should serve as guide in the selection of pesticides to use.

Larva control: Mosquito larvae are aquatic, deriving nourishment from organic matter in standing pools of water. Although this may be challenging to achieve over large areas as it requires the identification of larval pools, it can significantly reduce the reproductive rate of mosquitoes, keeping their numbers to a low level.

Larvicides are applied in identified pools containing mosquito larvae periodically. This can be expensive and requires a lot of manpower. Biological control methods can be used in larger pools of water, for example in rice paddy farms. Here, natural predators of mosquito larva are introduced. This is perhaps the most environmentally friendly method of mosquito control because no contaminants are introduced, and the indiscriminate killing seen with chemicals is avoided.

\section{Environmental Modification}

The modification of the natural ecosystem has provided a thriving environment for mosquitoes to breed and maintain high populations. Improper waste disposal, clogged drain ways, underground subways systems have been shown to be adequate for mosquito breeding. Farms 
are also excellent breeding sites for mosquitoes. These have not only provided good year-round breeding sites for mosquitoes, they have brought the large population of mosquitoes in close proximity to humans and their livestock. Environmental re-modifications can be implemented to reduce sites that mosquitoes can use for breeding.

Adequate waste disposal: The improper disposal of containers and used tires provide excellent breeding sites for mosquitoes. Developing an adequate waste collection and disposal system around human settlements and farms can reduce sites available for mosquitoes to breed.

Draining breeding sites: This is perhaps most important around human dwellings. Mosquitoes can breed in standing water in flower pots, wells, drainages and pools. These sites should be identified and eliminated on a regular basis.

Farming practices: Rice paddies are excellent breeding sites for mosquitoes and a major agricultural activity especially in rural areas. Plantations also breeding areas for mosquitoes. Introducing measures to reduce breeding and exposure of farmers to these vectors is important in reducing mosquito population and preventing transmission of the virus.

\section{Public Enlightenment and Behavioural changes}

Certain behaviours can determine the level of exposure to mosquito bites and possible transmission of the virus. Wearing protective clothing, eliminating breeding sites especially in flower pots, using insecticides in the home, clearing bushes around the home premises and the use of insecticide impregnated mosquito nets are behavioural changes that can be helpful in vector control and prevention of transmission. To achieve this, the public needs to be informed about the risks of the vectors and the benefits of the behavioural changes.

\section{Current Mosquito Control Strategies and Challenges in Malaysia}

Dengue virus, a flavivirus closely related to $\mathrm{WN}$ virus is endemic in Malaysia. A vector control programme targeting its mosquito vectors: Aedes aegypti and Aedes albopictus has been ongoing for years, applying a combination of measures including adult and larva control through use of chemicals, environmental management, house inspections and fogging [24]. The cost of this programme in 2010 was US\$73.5, representing $0.03 \%$ of the GDP for that year with a significant amount of directed at killing adult mosquitoes
[25]. A full evaluation of this control programmes needs to be carried out to determine its effectiveness at reducing mosquito populations. Although the vectors for Dengue and WN viruses are different and both mosquito species have different ecological preferences and feeding behaviours, measures aimed at eliminating a particular species of mosquitoes may be beneficial in controlling more than just the target species, since insecticides will kill all mosquitoes, available breeding sites will be reduced for al species, and behavioural adjustments and practices would mean a reduction in contact with the vectors. However, this isn't always true as a study in Macau showed that a decline in the population of Anopheles mosquitoes was followed by an increase in the population of Culex species [26]. A similar study on the effects of Aedes control on Culex population dynamics in Malaysia will be beneficial in implementing an integrated mosquito control programme. Concerns about the emergence of resistance to pesticides and the overall environmental impact of fogging needs to be investigated.

\section{Recommendations and Conclusion}

Available information suggests the presence and possible circulation of at least one strain of $\mathrm{WN}$ virus in mosquitoes, and antibody detection in birds and humans indicates exposure to the virus. Preventing an outbreak requires a well-designed strategy aimed at assessing the real risk of infection in livestock and humans and the control of vector population as well as preventing or minimizing contact between humans and the vectors. This can be achieved through strong cooperation between stake holders in human and veterinary health sectors, policy makers and the community.

Novel methods of vector control including the use of genetically modified mosquitoes that produce sterile progeny should be considered. This will eliminate the concerns about resistance to available pesticides and the environmental impact of using pesticide use.

An integrated mosquito surveillance and control system will provide information on mosquito populations, ecology and pathogens they harbour. This is essential for early detection and timely response to prevent an epidemic.

\section{References}

1. Gubler DJ (2007) The Continuing Spread of West Nile Virus in the Western Hemisphere. Clinical Infectious Diseases 45(8): 1039-1046. 


\section{Open Access Journal of Veterinary Science \& Research}

2. Caren Chancey, Andriyan Grinev, Evgeniya Volkova, Maria Rios (2015) The Global Ecology and Epidemiology of West Nile Virus. BioMed Research International, pp: 1-20.

3. Smithburn KC, Hughes TP, Burke AW, Paul JH (1940) A Neutrotropic Virus Isolated from the Blood of a Native of Uganda. American Journal of Tropical Medicine 20(4): 471-472.

4. Nash D, Mostashari F, Fine A, Miller J, O'Leary D, et al. (2001) The Outbreak of West Nile Virus Infection in the New York City Area in 1999. New England Journal of Medicine 344(24): 1807-1814.

5. Pesko KN, Ebel GD (2012) West Nile virus population genetics and evolution. Infection, Genetics and Evolution. Elsevier 12(2): 181-190.

6. Ching CY, Casals J, Bowen ET, Simpson DI, Platt GS, et al. (1970) Arbovirus infections in sarawak: The isolation of kunjin virus from mosquitoes of the culex pseudovishnui group. Annals of Tropical Medicine and Parasitology 64(3): 263-268.

7. Lanciotti RS, Roehrig JT, Deubel V, Smith J, Parker M, et al. (1999) Origin of the West Nile Virus Responsible for an Outbreak of Encephalitis in the Northeastern United States. Science 286(5448): 2333-2337.

8. Pond WL (1963) Arthropod-borne virus antibodies in sera from residents of South-East Asia. Transactions of the Royal Society of Tropical Medicine and Hygiene 57(5): 364-371.

9. Marlina S, Radzi SF, Lani R, Sieng KC, Rahim NF, et al. (2014) Seroprevalence screening for the West Nile virus in Malaysia's Orang Asli population. Parasites \& Vectors 7(1): 597.

10. Rais MN, Rahman Omar A, Abu J, Hussni Omar M (2011) Prevalence of West Nile Virus Antibody in Captive Bird Populations in Selected Areas in Selangor, Malaysia, pp: 127.

11. Liang G, Gao X, Gould EA (2015) Factors responsible for the emergence of arboviruses; strategies, challenges and limitations for their control. Emerging Microbes \& Infections 4(3): e18-e18.

12. Ciota AT (2017) West Nile virus and its vectors. Current Opinion in Insect Science. Elsevier 22: 28-36.
13. Londono-Renteria B, Colpitts TM (2016) A Brief Review of West Nile Virus Biology. West Nile Virus pp: 1-13.

14. Tonya M Colpitts, Michael J Conway, Ruth R Montgomery, Erol Fikrig (2012) West Nile Virus: biology, transmission, and human infection. Clinical Microbiology Reviews 25(4): 635-648.

15. Diamond MS (2009) Virus and Host Determinants of West Nile Virus Pathogenesis. PLoS Pathogens, 5(6): e1000452.

16. Grubaugh ND, Fauver JR, Rückert C, Weger-Lucarelli J, Garcia-Luna S, et al. (2017) Mosquitoes Transmit Unique West Nile Virus Populations during Each Feeding Episode. Cell Reports 19(4): 709-718.

17. Goudarz Molaei, Theodore G Andreadis, Philip M Armstrong, John F Anderson, Charles R Vossbrinck (2006) Host feeding patterns of Culex mosquitoes and West Nile virus transmission, northeastern United States. Emerging Infectious Diseases 12(3): 468-474.

18. Radrova J, Seblova V, Votypka J (2013) Feeding behavior and spatial distribution of Culex mosquitoes (Diptera: Culicidae) in wetland areas of the Czech Republic. Journal of Medical Entomology 50(5): 10971104.

19. Bruno Gomes, Carla A Sousa, José L Vicente, Leonor Pinho, Isabel Calderón, et al. (2013) Feeding patterns of molestus and pipiens forms of Culex pipiens (Diptera: Culicidae) in a region of high hybridization. Parasites \& Vectors 6(1): 93.

20. Megan L Fritz, Edward D Walker, Aaron J Yunker, Ian Dworkin (2014) Daily blood feeding rhythms of laboratory-reared North American Culex pipiens. Journal of Circadian Rhythms 12(1): 1.

21. Raymond M, Berticat C, Weill M, Pasteur N, Chevillon C (2001) Insecticide resistance in the mosquito Culex pipiens: what have we learned about adaptation? Genetica 113: 287-296.

22. Scott JG, Yoshimizu MH, Kasai S (2014) Pyrethroid resistance in Culex pipiens mosquitoes. Pesticide Biochemistry and Physiology 120: 68-76.

23. Tabbabi A, Daaboub J, Laamari A, Cheikh B, Jha I Ben, et al. (2017) Study Confirming Resistance to Fenitrothion of Culex pipiens (Diptera: Culicidae) from Central Tunisia. Hereditary Genet 6(1): 176. 


\section{Open Access Journal of Veterinary Science \& Research}

24. Song-Quan O (2016) Dengue Vector Control in Malaysia: A Review for Current and Alternative Strategies. Sains Malaysiana 45: 777-785.

25. Packierisamy PR, Ng CW, Dahlui M, Inbaraj J, Balan VK, et al. (2015) Cost of Dengue Vector Control Activities in Malaysia. The American Journal of Tropical Medicine and Hygiene 93(5): 1020-1027.
26. Easton ER (1994) Urbanization and Its Effects on the Ecology of Mosquitoes in Macau, Southeast Asia. Journal of the American Mosquito Control Association 10(4): 540-544. 